\title{
Etnobotânica na conservação de espécies com sementes sensíveis à dessecação: o exemplo da Eugenia brasiliensis Lam.
}

\author{
iD Edmir Vicente Lamarca ${ }^{1,4}$, (D) Clóvis José Fernandes de Oliveira Júnior² e (D) Claudio José Barbedoº \\ Recebido: 08.04.2019; aceito: 17.03.2020
}

Como citar: Lamarca, E.V., Oliveira Júnior, C.J.F., \& Barbedo, C.J. 2020. Etnobotânica na conservação de espécies com sementes sensíveis à dessecação: o exemplo da Eugenia brasiliensis Lam. Hoehnea 47: e372019. http://dx.doi. org/10.1590/2236-8906-37/2019

\begin{abstract}
Ethnobotany in the conservation of plant species with seeds susceptible to desiccation: the example of Eugenia brasiliensis Lam.). In this work, we aimed to demonstrate the importance of productive and/or economic strategies for plant biodiversity conservation programs, especially when the species produce recalcitrant seeds. A bibliographical survey was carried out and Eugenia brasiliensis Lam. (grumixameira) was used because of its desiccant sensitive seeds. Ethnobotanical aspects, food importance, and medicinal potentials were evaluated. The grumixameira has been used for food and medicinal purposes, being adequate for sustainable models of agricultural production. This species has excellent characteristics for the to strengthen its production chain, with the potential for profitability in the use of natural products derived from it. The adequacy of its use as a model to consolidate the productive-economic strategy is an important tool for preservation.
\end{abstract}

Keywords: agroecology, agroforestry systems, medicinal plants, plant biodiversity, recalcitrant seeds

RESUMO - (Etnobotânica na conservação de espécies com sementes sensíveis à dessecação: o exemplo da Eugenia brasiliensis Lam.). Procurou-se apresentar a importância da utilização de estratégias produtivas e/ou econômicas nos programas de conservação da biodiversidade vegetal, especialmente quando a espécie produz sementes recalcitrantes. Foi realizado levantamento bibliográfico e utilizou-se Eugenia brasiliensis Lam. (grumixameira) como modelo por suas sementes sensíveis à dessecação. Analisaram-se aspectos etnobotânicos, importância alimentar e seus potenciais medicinais. A grumixameira vem sendo utilizada para fins alimentares e medicinais, sendo bastante adequada aos modelos sustentáveis de produção agrícola. Essa espécie apresenta excelentes características para o fortalecimento de sua cadeia produtiva, com potencial de rentabilidade no uso dos produtos naturais dela derivados. A adequabilidade de seu uso como modelo para consolidar a estratégia produtivo-econômica faz-se uma importante ferramenta para a preservação.

Palavras-chave: agroecologia, biodiversidade vegetal, plantas medicinais, sementes recalcitrantes, sistemas agroflorestais

\section{Introdução}

O avanço da agricultura e urbanização sobre áreas de vegetação nativa cada vez mais exige o desenvolvimento de estratégias que permitam conservar, ex situ, as espécies em risco de extinção, ou mesmo as que podem entrar nessa categoria. Embora existam diversas formas para esse tipo de conservação, o armazenamento de sementes continua sendo a mais vantajosa, por agregar eficiência, economia, praticidade, entre outros fatores.
Muitas espécies apresentam sementes com características que permitam conservá-las por décadas, algumas vezes até séculos, ou seja, a tolerância à dessecação (Silva et al. 2018). Essa tolerância permite que as sementes sobrevivam à quase completa remoção de sua água, permitindo substancial redução nas atividades metabólicas tanto das sementes como de outros organismos associados a elas (Marco Filho 2015).

Contudo, há espécies que produzem sementes sensíveis à essa dessecação e, consequentemente, até que se desenvolva tecnologia apropriada para sua conservação

1 Universidade Ibirapuera, Avenida Interlagos, 1329, Chácara Flora, 04661-100 São Paulo, SP, Brasil

2 Instituto de Botânica de São Paulo, Núcleo de Pesquisa em Plantas Ornamentais, Avenida Miguel Stéfano, 3687, 04301-012 São Paulo, SP, Brasil

3 Instituto de Botânica de São Paulo, Núcleo de Pesquisa em Sementes, Avenida Miguel Stéfano, 3687, 04301-012 São Paulo, SP, Brasil 4 Autor para correspondência: edmirvicente18@gmail.com 
em armazenamento, outras formas de preservação da espécie devem ser utilizadas (Silva et al. 2018, Mayrinck et al. 2019, Stavrinides et al. 2019). Diante da tolerância à dessecação $\mathrm{e}$ armazenamento as sementes são popularmente classificadas como ortodoxas ou recalcitrantes, ou seja, respectivamente, tolerantes e intolerantes à dessecação. Fisiológica e cientificamente, porém, as sementes apresentam gradientes entre aquelas duas categorias, provavelmente decorrentes do estádio de maturação que se encontram quando são dispersas (Silva et al. 2018, Guevara-Ohara et al. 2019). Vale salientar, que aproximadamente $50 \%$ de espécies da flora tropical ou subtropical, como a Mata Atlântica, apresentam sementes sensíveis à dessecação (Tweddle et al. 2003).

O armazenamento de sementes em bancos de germoplasma é uma importante ferramenta para a conservação ex situ, sendo uma estratégia segura e economicamente viável, assegurando fontes de variabilidade genética, essenciais na construção de programas de produção e uso sustentável da flora nativa. Todavia, ainda que se tivesse tecnologia que permitisse armazenar as sementes sensíveis à dessecação por longos períodos, dificilmente se poderá concentrar, nos bancos de semente, toda a diversidade natural da espécie.

Uma forma de diminuir esse problema poderia ser, por exemplo, o intercâmbio de material das diferentes regiões, como algumas vezes ocorre na conservação on farm. Esta é definida como "a conservação de ecossistemas e habitats naturais e a manutenção e recuperação de populações viáveis de espécies em seu meio natural e, no caso de espécies domesticadas ou cultivadas, no meio em que desenvolveram suas propriedades distintas" (FAO, 1996). Esta estratégia de conservação permite o uso comercial sustentável, associado ao intercâmbio de sementes das populações locais, sejam elas nativas ou crioulas (Maharjan et al. 2011, Thomas et al. 2011, Sosinski Junior et al. 2019). Como envolve populações locais, esta estratégia de conservação é considerada in situ. Contudo, é possível pensar nesta forma de conservação utilizando não apenas populações locais, mas também populações e/ou espécies exóticas ao local, desde que considerados, também, os intercâmbios de material genético, o que caracterizaria uma conservação ex situ on farm. Parreiras (2003), em seu trabalho sobre a regulamentação dos jardins botânicos brasileiros, ilustra que, as estratégias de conservação implicam na combinação de políticas, ações, estruturação institucional e saberes diversos, dentre esses diversos saberes, podemos elucidar as diferentes estratégias de conservação in situ e ex situ e a Etnobotânica.

As formas mais eficientes de se conservar espécies ex situ, mas não em bancos de germoplasma (como o armazenamento de sementes ou o cultivo in vitro), envolvem o desenvolvimento de tecnologias que permitam que tal conservação seja incluída em um plano de aproveitamento econômico.

Portanto, além do investimento técnico e científico em estratégias de conservação e armazenamento das sementes, mostra-se de grande importância o resgate sobre os saberes tradicionais acerca do uso de plantas (Martin 2004), sendo fontes importantes de conhecimento para a construção de novas cadeias produtivas (Leonel 2000), as chamadas cadeias produtivas da sociobiodiversidade, que engloba saberes populares como usos medicinais, alimentares, madeireiros, artesanatos, utensílios, construções e outros.

A maneira de uso das plantas e o manejo racional dos recursos naturais, a preservação in locu do vegetal, o incentivo às atividades sustentáveis principalmente em países tropicais e subtropicais, onde as populações rurais dependem em parte das plantas e de seus produtos para sua subsistência e a valorização de uso dos produtos da biodiversidade local, fazem parte da ciência que estuda a relação entre as pessoas e as plantas, ou seja, a Etnobotânica (Diegues et al. 2000, Martin 2004, Gandolfo \& Hanazaki 2011). Além disso, a Etnobotânica se faz como um dos principais caminhos para a descoberta de produtos naturais bioativos (Maciel et al. 2002).

A agroecologia tem observado este conhecimento sistematizado pela Etnobotânica e utilizado para construção de propostas e modelos de produção e desenvolvimento rural sustentável, alavancando a utilização de espécies nativas em agroecossistemas sustentáveis. Apontando ainda que o uso da biodiversidade nativa aumenta o grau de resiliência nos sistemas de produção (Oliveira Junior \& Cabreira 2012, Oliveira Junior et al. 2013). Desta forma, a inserção de espécies nativas, em cadeias produtivas ou em sistemas de produção pode ser uma interessante estratégia de conservação.

As florestas tropicais e subtropicais do Brasil apresentam espécies de grande importância tanto econômica quanto ecológica, como as do gênero Eugenia, representado por mais de 100 espécies e pertencente à família Myrtaceae (Gressler et al. 2006, Romagnolo \& Souza 2006, Lamarca et al. 2013).

A grumixameira (Eugenia brasiliensis Lam.) é uma espécie arbórea, endêmica do Brasil, de Mata Atlântica, pertencente à família Myrtaceae e ocorre na faixa litorânea da Bahia até Santa Catarina (Corrêa 1984, Flora do Brasil 2020 em construção, 2018). Sua distribuição geográfica têm ocorrências já confirmadas no Nordeste (Bahia), no Sudeste (Espírito Santo, Minas Gerais, Rio de Janeiro e São Paulo) e no Sul (Paraná e Santa Catarina). Presente no domínio fitogeográfico de Mata Atlântica, em vegetações do tipo Floresta Estacional Semidecidual, Floresta Ombrófila, Floresta Ombrófila Mista e Restinga (Flora do Brasil 2020 em construção, 2018). Sua madeira é branca, porém não mole, sendo, no passado, muito utilizada em obras de torno e marcenaria (Corrêa 1984), fato que contribuiu para redução de suas populações em áreas de florestas naturais. Foi utilizada para produção madeireira no estado de São Paulo, por seu relativo rápido crescimento, e hoje em dia é bastante comum em projetos de restauração ecológica. Suas folhas são aromáticas e adstringentes, devido ao alto teor 
de taninos, e apresenta uso popular como antirreumática e diurética (Corrêa 1984). Além disso, suas folhas apresentam uma ampla variedade química de óleos essências, já isolados e identificados (Lima et al. 2008).

A grumixameira produz frutos denominados "grumixamas". O termo grumixama já sofreu várias flexões em sua grafia, inicialmente era escrito como "Koximã", como registrado por Navarro (2013). Grumixameira e grumixama são termos encontrados, também, em obras da literatura brasileira, como em "Corografia Brazílica" de Manuel Aires de Casal de 1817 e "O suplício de um marido" de Ferreira Leal de 1888 (Cunha 1998). As grumixamas, amarelas ou roxas, são saborosas, com bom potencial tanto para consumo in natura como processados na forma de sucos, geleias ou sorvetes, além de serem alimentos para diversas aves e mamíferos. Já existe no mercado a geleia de grumixama, porém, ainda sem cadeia produtiva estruturada e de modo bastante inicial, e mesmo a fabricação de sorvete de grumixama ainda fica restrita a pequenas sorveterias artesanais.

Devido às características de suas sementes, raramente descritas na literatura, as grumixameiras apresentam-se como interessantes materiais para estudos fisiológicos e, com isso, muitos foram os avanços nos estudos com suas semente, tais como, o potencial de regeneração dos embriões (Silva et al. 2005), as diferenças nos limites de tolerância à dessecação e armazenamento (Kohama et al. 2006, Delgado \& Barbedo 2007, Delgado \& Barbedo 2012), a plasticidade na exigência térmica para a germinação (Lamarca et al. 2011), o uso de tecnologias de sementes para análise do vigor e viabilidade (Lamarca \& Barbedo 2014), a germinação em diferentes substratos (Tomielis et al. 2016), os tratamentos osmóticos e térmicos no controle de fungos associados às sementes (Françoso \& Barbedo 2016), entre outros. Todavia, mesmo com os avanços científicos em sementes desta espécie, ainda são grandes as dificuldades de seu armazenamento, uma vez que suas sementes são sensíveis à dessecação e ao armazenamento (Kohama et al. 2006, Delgado \& Barbedo 2007, Delgado \& Barbedo 2012).

Diante do exposto, com o desmatamento e as ameaças às florestas tropicais e subtropicais do Brasil, como a Mata Atlântica, torna de grande importância registrar a dificuldade de armazenamento de sementes nativas da Mata Atlântica sensíveis à dessecação, como, por exemplo, a grumixameira (Eugenia brasiliensis Lam.). Além disso, valorizar e ressaltar os potenciais de uso econômico da espécie, propondo diversas estratégias que ampliem a sua possibilidade de conservação. Assim, no presente estudo, por meio de revisão da literatura, serão apresentadas informações sobre a grumixameira (Eugenia brasiliensis Lam.), ou seja, estudos sobre a sensibilidade à dessecação de suas sementes, registros etnobotânicos e os diversos usos da espécie para utilização econômica, com descrição de seus potenciais medicinais, alimentares e outros, com intuito de apontar estratégias para a conservação e preservação da espécie.

\section{Material e Métodos}

Para a análise da sensibilidade à dessecação de sementes de grumixameira (Eugenia brasiliensis Lam.) os dados foram obtidos dos seguintes estudos: Kohama et al. (2006), Delgado \& Barbedo (2007) e Lamarca \& Barbedo (2014). De cada estudo, foram apresentados os dados de teor de água, geminação e desenvolvimento de plântulas normais de sementes de grumixameira com diferentes níveis de secagem. Assim, no presente estudo, estes resultados acima foram organizados em quatro níveis de secagem, ou seja, sementes sem secagem, primeiro nível de secagem, segundo nível de secagem e terceiro nível de secagem.

Os registros etnobotânicos e medicinais de grumixameira foram obtidos por meio de levantamento da bibliografia científica disponível nos portais "Google Acadêmico" (https://scholar.google.com.br) e "SciELO" (http://www.scielo.org/php/index.php/). Para tanto, foram utilizadas as seguintes palavras-chave: Eugenia brasiliensis, grumixama, grumixameira, Etnobotânica, alimentar, medicinal, madeirável, ornamental, antioxidante, anti-inflamatória, antibacteriana. O período pesquisado foi entre 2006 e 2018. Os critérios de inclusão para seleção dos registros científicos foram: ser artigo científico, nota técnica ou científica, resumo expandido, teses ou dissertações, período de publicação pesquisado, presença dos termos nos conteúdos das publicações e ser nos idiomas português e inglês. Os critérios de exclusão para seleção dos registros científicos foram: não ser artigo científico, nota técnica ou científica, resumo expandido, teses ou dissertações, não estar dentro do período de publicação pesquisado, não ter a presença dos termos nos conteúdos das publicações e não ser nos idiomas português e inglês (Lamarca et al. 2013).

Os dados obtidos foram quantificados e agrupados quanto ao tipo de uso de grumixameira e quanto às estruturas utilizadas da planta. Os dados referentes ao tipo de uso foram organizados nas seguintes categorias: alimentar, medicinal, madeirável e ornamental. Os dados referentes às estruturas utilizadas foram organizados nas seguintes categorias: caule, folha, flor e fruto (Lamarca et al. 2013). Com esses dados supracitados, ou seja, tipo de uso e estruturas utilizadas da planta calculou-se distribuições de frequências. Metodologia do cálculo de distribuição de frequência, adaptada de Ribeiro Júnior (2004). Já as informações sobre a importância e curiosidades da espécie e informações fitoquímicas, farmacológicas, ecológicas, fisiológicas e germinativas foram obtidas principalmente de artigos científicos e dicionários históricos da língua Tupi.

\section{Resultados e Discussão}

Sensibilidade à dessecação de sementes de grumixameira Os dados obtidos por Kohama et al. (2006), Delgado \& Barbedo (2007) e Lamarca \& Barbedo (2014) mostram queda 
gradativa na germinação e desenvolvimento de plântulas normais com o aumento do nível de secagem (tabela 1), demonstrando a sensibilidade à dessecação das sementes de grumixameira. Em todos os estudos, independente da origem do material, da época dos experimentos e do biótipo ou cor dos frutos (amarelo ou roxo), as sementes se mostraram sensíveis quando o teor de água é reduzido para valores abaixo de $40 \%$.

Esses resultados indicam a dificuldade no armazenamento dessas sementes e, consequentemente, na sua conservação ex situ. Essa característica é comum a outras espécies do mesmo gênero, como Eugenia cerasiflora, Eugenia involucrata, Eugenia pyriformis, Eugenia umbeliflora, Eugenia uniflora (Delgado \& Barbedo 2007, Lamarca \& Barbedo 2014). O grau de sensibilidade à dessecação, contudo, é dependente não apenas da espécie, mas também das condições ambientais nas quais as sementes foram formadas (Lamarca et al. 2016).

Ao longo dos últimos anos, muitos foram os avanços científicos acerca da tolerância à dessecação de sementes $\mathrm{e}$ são de extrema importância para o entendimento da biologia vegetal e para o desenvolvimento de tecnologias para a conservação ex situ por meio de bancos de sementes ou de germoplasma. Todavia, ainda é grande a dificuldade de conservação de espécies com sementes sensíveis à dessecação por meio de bancos de sementes, como a grumixameira, tornando ainda mais importante a utilização de estratégias e ferramentas técnicas e científicas da Etnobotânica.

Etnobotânica e uso econômico e medicinal da grumixameira - Na literatura científica, o uso da grumixameira é predominantemente medicinal, mas também são registrados outros usos, como alimentar e exploração de madeira (tabela 2). A parte da planta mais utilizada é a folha, seguida do fruto e do caule (tabela 2).

Quando analisados os dados por meio da distribuição de frequência, verifica-se uma grande amplitude de frequência entre os usos e as estruturas utilizadas de grumixameiras (figura 1). Ou seja, para o tipo de uso, verifica-se 53\% para o uso medicinal, $41 \%$ para o uso alimentar e $6 \%$ para o uso madeirável (figura 1a). Já para as estruturas utilizadas, verifica-se $44 \%$ para a folha, $38 \%$ para o fruto e $18 \%$ para o caule (figura $1 b$ ). Não se verificaram registros para o uso ornamental e das flores.

A grumixameira é importante em projetos de recuperação de áreas degradadas e vem aumentando seu uso na arborização urbana, numa proposta educativa e conscientizadora sobre a importância da flora local e seu conhecimento pela população, adotadas por tendências atuais de paisagismo e urbanismo no eixo de cidades sustentáveis (Oliveira Junior et al. 2013). São ainda excelentes opções para produção em sistemas agroflorestais e para compor quintais e pomares florestais (Lamarca et al. 2013).

Variadas propriedades medicinais e farmacêuticas foram comprovadas cientificamente para Eugenia brasiliensis (tabela 3), com diversos potenciais apresentados em suas folhas, cascas do caule e frutos (Lamarca et al. 2013, Queiroz et al. 2015). O conhecimento tradicional pode ser o principal caminho para a descoberta de novos produtos naturais, contudo, é importante enfatizar que o conhecimento sobre o uso de plantas medicinais, muitas vezes, é grande sob o ponto de vista empírico e restrito sob o ponto de vista científico.

Como pode ser observados na tabela 3 , estudos demonstram a atividade anti-inflamatória de Eugenia brasiliensis (Ramos et al. 2006, Pietrovski et al. 2008, Siebert et al. 2017); a atividade antibiótica também é encontrada na literatura científica (Benfatti et al. 2010, Magina et al. 2012, Rodrigues et al. 2016). O potencial antioxidante da grumixameira também é apontado em alguns estudos (Magina et al. 2010). A grumixama apresenta um teor considerável de vitamina C (Arévalo et al. 2014), o que estaria de acordo com a atividade antioxidante citada acima. As distintas colorações dos frutos, que caracterizam as grumixameiras em variedades ou biótipos distintos apresentam também óleos essenciais com diferentes composições (Moreno et al. 2007). A composição e variação química de óleos essenciais presentes nas folhas de Eugenia brasiliensis também foram registrados na literatura científica (Lima et al. 2008), assim como, os efeitos antidepressivos de Eugenia brasiliensis (Colla et al. 2012).

Outros estudos demonstraram os potenciais medicinais de espécies de Eugenia, incluído a Eugenia brasiliensis, como o realizado por Queiroz et al. (2015), que fizeram uma revisão da literatura referente às características botânicas, etnobotânicas, atividades farmacológicas, toxicológicas e a constituição química de espécies de Eugenia. E o realizado por Lamarca et al. (2013), que analisaram os potenciais alimentares e medicinais de espécies do gênero Eugenia, a partir de registros da literatura científica sobre o conhecimento tradicional ou local.

Atividades produtivas sustentáveis são alternativas econômicas que visam valorizar os produtos da biodiversidade vegetal, promovendo uma relação direta entre geração de renda e conservação ambiental (Queiroz 2005). É de grande importância salientar sobre a necessidade de criação de políticas públicas, legislações, instituições e centros de desenvolvimento que protejam a biodiversidade e o conhecimento tradicional, vislumbrando evitar assim, a biopirataria, uma vez que sistemas de patentes podem colocar em risco os recursos genéticos, não proporcionando nenhum retorno financeiro para os pontos de origem de tais conhecimentos (Oliveira Junior et al. 2012, Agostinho 2016).

Como constatado, a grumixameira apresenta uma diversidade de usos com distintas possibilidades para produção, que vai dos frutos in natura ou processados, de folhas para indústria farmacêutica e de fitoterápicos, e mesmo como produção madeireira, o que é bastante interessante para o produtor rural, pois aumentam as possibilidades de produtos e obtenção de renda. A 
Tabela 1. Teor de água, germinação, desenvolvimento de plântulas normais de sementes de grumixameira (Eugenia brasiliensis Lam.) submetidas a diferentes níveis de secagem. Dados obtidos das seguintes referências: Kohama et al. (2006), Delgado \& Barbedo (2007) e Lamarca \& Barbedo (2014).

Table 1. Water content, germination, development of normal seedlings from seeds of grumixameira (Eugenia brasiliensis Lam.) subjected to different drying levels. Data obtained from the following references: Kohama et al. (2006), Delgado \& Barbedo (2007) and Lamarca \& Barbedo (2014).

\begin{tabular}{lccc}
\hline Níveis de secagem & $\begin{array}{c}\text { Teor de água } \\
(\%)\end{array}$ & $\begin{array}{c}\text { Germinação } \\
(\%)\end{array}$ & $\begin{array}{c}\text { Plântulas normais } \\
(\%)\end{array}$ \\
\hline
\end{tabular}

Kohama et al. (2006)

(Biótipo roxo)

Sem secagem

Primeiro nível de secagem

Segundo nível de secagem

Terceiro nível de secagem

Kohama et al. (2006)

(Biótipo amarelo)

Sem secagem

Segundo nível de secagem

Terceiro nível de secagem

Delgado \& Barbedo (2007) (Biótipo roxo)

Sem secagem

Primeiro nível de secagem

Segundo nível de secagem

Terceiro nível de secagem

Delgado \& Barbedo (2007) (Biótipo amarelo)

Sem secagem

Primeiro nível de secagem

Segundo nível de secagem

Terceiro nível de secagem

Lamarca \& Barbedo (2014) (Biótipo roxo)

Sem secagem

Primeiro nível de secagem

Segundo nível de secagem

Terceiro nível de secagem
$98 \quad 94$

$98 \quad 92$

$92 \quad 88$

$65 \quad 56$ 
Tabela 2. Registros etnobotânicos e medicinais de grumixameira (Eugenia brasiliensis Lam.) obtidos por meio de revisão da bibliografia científica. Tipo de uso, estrutura utilizada da planta, total de registros e referências bibliográficas. Período pesquisado de 2006 a 2018.

Table 2. Ethnobotanical and medicinal records of grumixameira (Eugenia brasiliensis Lam.) obtained through a review of the scientific literature. Type of use, plant structure used, total number of records, and bibliographical references. Search period from 2006 to 2018.

\begin{tabular}{lccc}
\hline Tipo de Uso & Total de Registros & Estrutura Utilizada & Total de Registros \\
\hline Alimentar & 7 & Caule & 3 \\
Medicinal & 9 & Folha & 7 \\
Madeirável & 1 & Fruto & 6 \\
Ornamental & 0 & Flor & 0 \\
\hline
\end{tabular}

Referências Bibliográficas: Ramos et al. (2006), Pietrovski et al. (2008), Borges \& Peixoto (2009), Pilla \& Amorozo (2009), Benfatti et al. (2010), Magalhães (2010), Magina et al. (2010), Colla et al. (2012), Magina et al. (2012), Arévalo et al. (2014), Lunelli et al. (2016), Pereira et al. (2016), Rodrigues et al. (2016), Siebert et al. (2017), Villaça \& Magenta (2017), Leal et al. (2018), Machado \& Boscolo (2018).

Tabela 3. Atividades medicinais de diferentes estruturas de grumixameira (Eugenia brasiliensis Lam.). Informações obtidas por meio de revisão bibliográfica.

Table 3. Scientific records of medicinal activities of different structures of grumixameira (Eugenia brasiliensis Lam.). Information obtained through the bibliographic review.

\begin{tabular}{lcc}
\hline Atividades & Estruturas Utilizadas & Referências Bibliográficas \\
\hline Anti-inflamatória & Folhas & Ramos et al. (2006), Pietrovski et al. (2008), Siebert et al. (2017) \\
Antibacteriana & Folhas, Frutos & Benfatti et al. (2010), Magina et al. (2012), Rodrigues et al. (2016) \\
Antioxidante & Caule, Folhas, Frutos & Magina et al. (2010), Arévalo et al. (2014) \\
Antidepressiva & Folhas & Colla et al. (2012) \\
\hline
\end{tabular}

espécie mostra-se, portanto, com grande potencial para o desenvolvimento de novas cadeias produtivas, também chamadas de cadeias produtivas da sociobiodiversidade. É assim, ótima opção para produção da agricultura familiar, que trabalha com produção mais diversificada, sem deixar de sê-la, no entanto, interessante para produção em larga escala em grandes fazendas.

Cada vez mais modelos e sistemas de agricultura ecológica, entre eles os sistemas agroflorestais e de policultivos, têm sido apontados como apropriados para mitigação aos efeitos das mudanças climáticas e efeito estufa e de grande auxílio na conservação da biodiversidade (Bhagwat et al. 2008, Luedeling et al. 2014), e maior resiliências aos agroecossistemas e na prestação de serviços ambientais (Castro et al. 2009, Jose 2009), sendo também apontados como alternativas econômicas viáveis de empreendimento rural (Santos \& Paiva 2002, Ramos et al. 2009). A utilização da biodiversidade em sistemas produtivos sustentáveis vem sendo apontada de grande valor para conservação dos recursos naturais (Jackson et al. 2007, Jose 2012). Por fim, o conhecimento da grumixameira, subsidiado pela Etnobotânica, mostra o grande potencial econômico e medicinal desta espécie, podendo ser uma interessante fonte de renda em sistemas agroflorestais, para agricultura familiar e comunidades tradicionais, vislumbrando a conservação e preservação da espécie e da biodiversidade.

Com este diálogo entre diferentes abordagens científicas (etnobotânica, agroecologia e fisiologia de sementes), são oferecidos subsídios para ampliar as estratégias de conservação dessa importante espécie nativa do Brasil, igualmente da Floresta Atlântica e da biodiversidade vegetal. Enfatizando-se a dificuldade de armazenamento das sementes, decorrente do seu grau de sensibilidade à dessecação, e demonstrando seu uso por comunidades tradicionais, em sistemas agroflorestais, pomares e quintais florestais, conforme registros etnobotânicos da literatura.

Demonstra-se aqui, o grande potencial conservacionista que a associação dessas áreas apresenta para a preservação da biodiversidade vegetal com a inclusão das espécies em cadeias produtivas. Essas práticas, associadas às já existente do intercâmbio de materiais entre as comunidades (caracterizando a conservação on farm), poderão garantir 

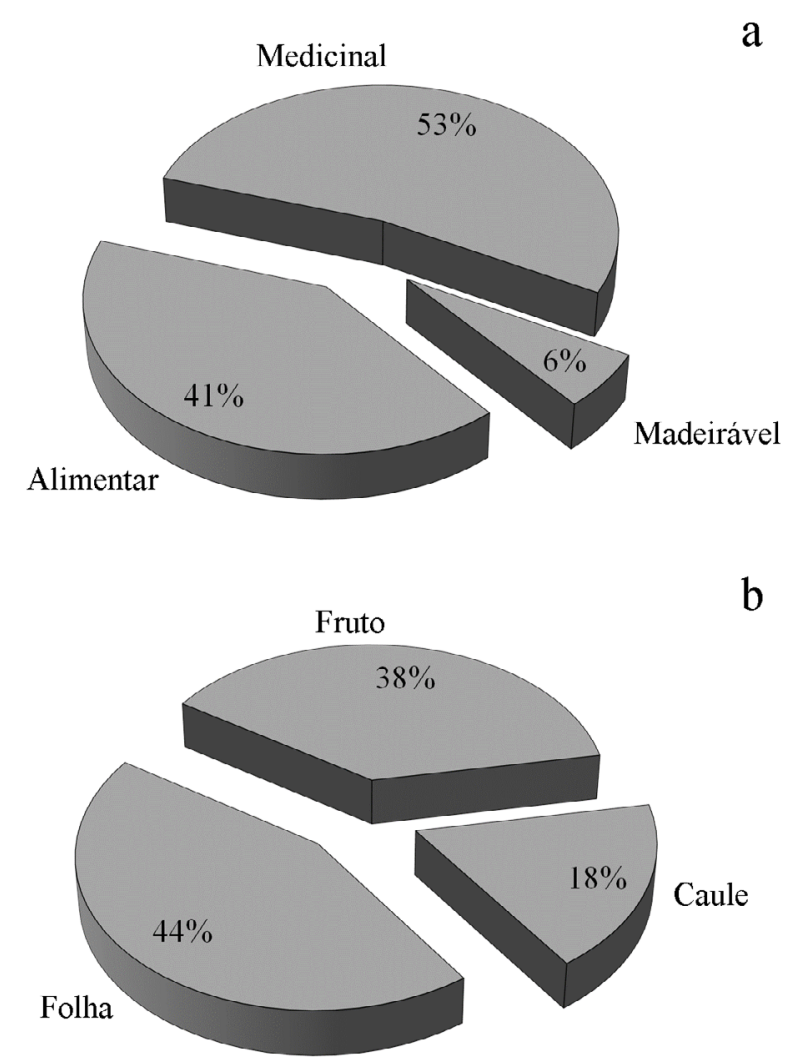

Figura 1. Distribuição de frequência de registros etnobotânicos e medicinais de grumixameiras (Eugenia brasiliensis Lam.). a. Tipo de uso. b. Estrutura utilizada da planta. Dados obtidos por meio de revisão bibliográfica.

Figure 1. Frequency distribution of ethnobotanical and medicinal records of grumixameira (Eugenia brasiliensis Lam.). a. Type of use. b. plant structure used. Data obtained through the bibliographic review.

o aproveitamento sustentável, até mesmo evitando o risco de extinção de diversas espécies. Ressalta-se, ainda, o potencial que a grumixameira apresenta para compor modelos de agricultura ecológica, que são capazes de prestar serviços ambientais, além da produção. Assim, conhecer uma espécie vegetal em seus diferentes aspectos, ou seja, botânicos, históricos, etnobotânicos, físiológicos, ecológicos, fitoquímicos, farmacológicos, entre outros, é um importante caminho para sua preservação, bem como para a conservação do patrimônio natural de uma nação, a biodiversidade vegetal.

\section{Literatura citada}

Agostinho, A.B. 2016. Centro de investigação e de desenvolvimento em etnobotânica: transformando o conhecimento tradicional em científico. Biodiversidade 15: 67-76.
Arévalo, R.P., Servignini, L.P., Silva, L.A.da., Maldonado, C.A.B., Arthur, V. \& Arevalo-Pinedo, A. 2014. Concentração de minerais presentes em frutos da grumixameira (Eugenia brasiliensis Lam.). Cadernos de Agroecologia 9: 1-9.

Benfatti, C.S., Cordova, S.M.de., Guedes, A., Magina, M.D.A. \& Cordova, C.M.M.de. 2010. Atividade antibacteriana in vitro de extratos brutos de espécies de Eugenia sp frente a cepas de molicutes. Revista PanAmazônica de Saúde 1: 33-39.

Bhagwat, A.S., Wills, K.J. Birks, J.B. \& Whitaker, R.J. 2008. Agroforestry: a refuge for tropical biodiversity? Trends in Ecology and Evolution 23: 261-267.

Borges, R. \& Peixoto, A.L. 2009. Conhecimento e uso de plantas em uma comunidade caiçara do litoral sul do Estado do Rio de Janeiro, Brasil. Acta Botanica Brasilica 23: 769-779.

Castro, A.P., Fraxe, T.J.P., Santiago, J.L., Matos, R.B. \& Pinto, I.C. 2009. Os sistemas agroflorestais como alternativa de sustentabilidade em ecossistemas de várzea no Amazonas. Acta Amazonica 39: 279-288.

Colla, A.R.S., Machado, D.G., Bettio, L.E.B., Colla, G., Magina, M.D.A., Brighente, I.M.C. \& Rodrigues, A.L.S. 2012. Involvement of monoaminergic systems in the antidepressant-like effect of Eugenia brasiliensis Lam. (Myrtaceae) in the tail suspension test in mice. Journal of Ethnopharmacology 143: 720-731.

Corrêa, M.P. 1984. Dicionário das plantas úteis do Brasil e das exóticas cultivadas. Volume III. Imprensa Nacional, Rio de Janeiro.

Cunha, A.G.da. 1998. Dicionário histórico das palavras portuguesas de origem tupi. 4 ed. Companhia Melhoramentos, São Paulo, Universidade de Brasília, Brasília.

Delgado, L.F. \& Barbedo, C.J. 2012. Water potential and viability of seeds of Eugenia (Myrtaceae), a tropical tree species, based upon different levels of drying. Brazilian Archives of Biology and Technology 55: 583-590.

Delgado, L.F. \& Barbedo, C.J. 2007. Tolerância à dessecação de sementes de espécies de Eugenia. Pesquisa Agropecuária Brasileira 42: 265-272.

Diegues, A.C., Arruda, R.S.V., Silva, V.C.F., Figols, F.A.B. \& Andrade, D. 2000. Os saberes tradicionais e a biodiversidade no Brasil. MMA/COBIO, Brasília/ NUPAUB-USP, São Paulo.

FAO. 1996. The state of the world's plant genetic resources for food and agriculture. Food and Agriculture Organization of the United Nations, Roma, Itália.

Flora do Brasil 2020 em construção. Jardim Botânico do Rio de Janeiro. Disponível em http://floradobrasil. jbrj.gov.br/reflora/floradobrasil/FB10359 (acesso em 21-XII-2018). 
Françoso, C.F. \& Barbedo, C.J. 2016. Osmotic and heat treatments on control of fungi associated with seeds of Eugenia brasiliensis and E. pyriformis (Myrtaceae). Journal of Seed Science 38: 195-203.

Gandolfo, E.S. \& Hanazaki, N. 2011. Etnobotânica e urbanização: conhecimento e utilização de plantas de restinga pela comunidade nativa do distrito do Campeche (Florianópolis, SC). Acta Botânica Brasílica 25: 168-177.

Gressler, E., Pizo, M.A. \& Morellato, P.C. 2006. Polinização e dispersão de sementes em Myrtaceae do Brasil. Revista Brasileira de Botânica 29: 509-530.

Guevara-Ohara, J.E., Cardozo-Conde, C.I. \& SantosMeléndez, L.G. 2019. Tolerancia a la desecación y almacenamiento de la semilla de guayaba (Psidium guajava). Agronomía Costarricense 43: 107-121.

Jackson, L.E., Pascual, U. \& Hodgkin, T. 2007. Utilizing and conserving agrobiodiversity in agricultural landscapes. Agriculture, Ecosystems \& Environment 121: 196-210.

Jose, S. 2009. Agroforestry for ecosystem services and environmental benefits: an overview. Agroforestry Systems 76: 1-10.

Jose, S. 2012. Agroforestry for conservation and enhancing biodiversity. Agroforestry Systems 85: 1-8.

Kohama, S., Maluf, A.M., Bilia, D.A.C. \& Barbedo, C.J. 2006. Secagem e armazenamento de sementes de Eugenia brasiliensis Lam. (grumixameira). Revista Brasileira de Sementes 28: 72-78.

Lamarca, E.V. \& Barbedo, C.J. 2014. Methodology of the tetrazolium test for assessing the viability of seeds of Eugenia brasiliensis Lam., Eugenia uniflora L. and Eugenia pyriformis Cambess. Journal of Seed Science 36: 427-434.

Lamarca, E.V., Baptista, W., Rodrigues, D.S. \& Oliveira Júnior, C.J.F.de. 2013. Contribuições do conhecimento local sobre o uso de Eugenia spp. em sistemas de policultivos e agroflorestais. Revista Brasileira de Agroecologia 8: 119-130.

Lamarca, E.V., Camargo, M.B.P.de., Teixeira, S.de.P., Silva, E.A.A.da., Faria, J.M.R. \& Barbedo, C.J. 2016. Variations in desiccation tolerance in seeds of Eugenia pyriformis: dispersal at different stages of maturation. Revista Ciência Agronômica 47: 118-126.

Lamarca, E.V., Silva, C.V. \& Barbedo, C.J. 2011. Limites térmicos para a germinação em função da origem de sementes de espécies de Eugenia (Myrtaceae) nativas do Brasil. Acta Botanica Brasilica 25: 293-300.

Leal, M.L., Alves, R.P. \& Hanazaki, N. 2018. Knowledge, use, and disuse of unconventional food plants. Journal of Ethnobiology and Ethnomedicine 14: 1-9.

Leonel, M. 2000. Bio-sociodiversidade: preservação e mercado. Estudos Avançados 14: 321-346.

Lima, N.P., Cerqueira, S.H.F., Fávero, O.A., Romoff, P. \& Lago, J.H.G. 2008. Composition and chemical variation of the essential oil from leaves of Eugenia brasiliensis Lam. And Eugenia sp. (Myrtaceae). Journal of Essential Oil Research 20: 223-225.
Luedeling, E., Kindt, R., Huth, N. \& Koenig, K. 2014. Agroforestry systems in a changing climate - challenges in projecting future performance. Current Opinion in Environmental Sustainability 6: 1-7.

Lunelli, N.P., Ramos, M.A. \& Oliveira Júnior, C.J.F.de. 2016. Do gender and age influence agroforestry farmers' knowledge of tree species uses in an área of the Atlantic Forest, Brazil? Acta Botanica Brasilica 30: 667-682.

Machado, C.de.C. \& Boscolo, O.H. 2018. Plantas alimentícias não convencionais em quintais da comunidade da Fazendinha, Niterói, Rio de Janeiro, Brasil. Revista Brasileira de Biociências 16: 28-36.

Maciel, M.A.M., Pinto, A.C., Veiga Júnior, V.F., Grynberg, N.F. \& Echevarria, A. 2002. Plantas medicinais: a necessidade de estudos multidisciplinares. Química Nova 25: 429-438.

Magalhães, A.C. 2010. Etnobotânica, saberes locais e agricultura no contexto de uma floresta urbana: maciço da Pedra Branca, RJ. Dissertação de Mestrado, Pontifícia Universidade Católica do Rio de Janeiro, Rio de Janeiro.

Magina, M.A., Gilioli, A., Moresco, H.H., Colla, G., Pizzolatti, M.G. \& Brighente, I.M.C. 2010. Atividade antioxidante de três espécies de Eugenia (Myrtaceae). Latin American Journal of Pharmacy 29: 376-82.

Magina, M.D.A., Dalmarco, E.M., Dalmarco, J.B., Colla, G., Pizzolatti, M.G. \& Brighente, I.M.C. 2012. Bioactive triterpenes and phenolics of leaves of Eugenia brasiliensis. Química Nova 35: 1184-1188.

Maharjan, S.K., Gurung, A.R. \& Sthapit, B.R. 2011. Enhancing on-farm conservation of agro-biodiversity through community seed bank: an experience of Western Nepal. The Journal of Agricultural and Environment 12: 132-139.

Marcos Filho, J. 2015. Fisiologia de sementes de plantas cultivadas. Abrates, Londrina.

Martin, G.J. 2004. Ethnobotany: a methods manual. Earthscan, London.

Mayrinck, R.C., Vilela, L.C., Pereira, T.M., RodriguesJunior, A.G., Davide, A.C. \& Vaz, T.A.A. 2019. Seed desiccation tolerance/sensitivity of tree species from Brazilian biodiversity hotspots: considerations for conservation. Trees 33: 777-785.

Moreno, P.R.H., Lima, M.E.L., Sobral, M., Young, M.C.M., Cordeiro, I., Apel, M.A., Limberger, R.P. \& Henriques, A.T. 2007. Essential oil composition of fruit colour varieties of Eugenia brasiliensis Lam. Scientia Agricola 64: 428-432.

Navarro, E.A. 2013. Dicionário de tupi antigo: a língua indígena clássica do Brasil. 1 ed. Global, São Paulo.

Oliveira Junior, C.J.F.de. \& Cabreira, P.P. 2012. Sistemas agroflorestais: potencial econômico da biodiversidade vegetal a partir do conhecimento tradicional ou local. Revista Verde de Agroecologia e Desenvolvimento Sustentável 7: 212-224. 
Oliveira Junior, C.J.F.de., Cabreira, P.P. \& Begossi, A. 2012. The dilemma of plant knowledge and compensation for native people living in brazilian biomes. Journal of Ecosystem \& Ecography 2: 100-108.

Oliveira Junior, C.J.F.de., Gonçalves, F.S., Couto, F. \& Matajs, L. 2013. Potencial das espécies nativas na produção de plantas ornamentais e paisagismo agroecológico. Revista Brasileira de Agroecologia 8: 190-200.

Oliveira Junior, C.J.F.de., Voigtel, S.D.S., Nicolau, S.A. \& Aragaki, S. 2018. Sociobiodiversidade e agricultura familiar em Joanópolis, SP, Brasil: potencial econômico da flora local. Hoehnea 45: 40-54.

Parreiras, O.M.U.de.S. 2003. A regulamentação dos jardins botânicos brasileiros: ampliando as perspectivas de conservação da biodiversidade. Rodriguésia 54: 33-54.

Pereira, G.de.S., Noelli, F.S., Campos, J.B., Santos, M.P. \& Zocche, J.J. 2016. Ecologia histórica guarani: as plantas utilizadas no bioma Mata Atlântica do litoral sul de Santa Catarina, Brasil (parte 1). Cadernos do LEPAARQ 13: 197-246.

Pietrovski, E.F., Magina, M.D.A., Gomig, F., Pietrovski, C.F., Micke, G.A., Barcellos, M., Pizzolatti, M.G., Cabrini, D.A., Brighente, I.M.C. \& Otuki, M.F. 2008. Topical anti-inflammatory activity of Eugenia brasiliensis Lam. (Myrtaceae) leaves. Journal of Pharmacy and Pharmacology 60: 479-487.

Pilla, M.A.C. \& Amorozo, M.C.de.M. 2009. O conhecimento sobre os recursos vegetais alimentares em bairros rurais no Vale do Paraíba, SP, Brasil. Acta Botanica Brasilica 23: 1190-1201.

Queiroz, H.L. 2005. A reserva de desenvolvimento sustentável Mamirauá. Estudos Avançados 19: 183-203.

Queiroz, J.M.G., Suzuki, M.C.M., Motta, A.P.R., Nogueira, J.M.R. \& Carvalho, E.M.de. 2015. Aspectos populares e científicos do uso de espécies de Eugenia como fitoterápico. Revista Fitos 9: 73-159.

Ramos, M.F.S., Siani, A.C., Souza, M.C., Rosas, E.C. \& Henriques, M.G.M.O. 2006. Avaliação da atividade antiinflamatória dos óleos essenciais de cinco espécies de Myrtaceae. Revista Fitos 2: 58-66.

Ramos, S.F., Chabaribery, D., Monteiro, A.V.V. \& Silva, J.R. 2009. Sistemas agroflorestais: estratégia para preservação ambiental e geração de renda aos agricultores familiares. Informações Econômicas 39: 37-48.

Ribeiro Júnior, J.I. 2004. Análise estatística no Excel: guia prático. Universidade Federal de Viçosa (UFV), Viçosa.

Rodrigues, A.C., Oliveira, B.D.de., Silva, E.R.da., Sacramento, N.T.B., Bertoldi, M.C. \& Pinto, U.M. 2016. Anti-quorum sensing activity of phenolic extract from Eugenia brasiliensis (Brazilian cherry). Food Science and Technology 36: 337-343.
Romagnolo, M.B. \& Souza, M.C. 2006. O gênero Eugenia L. (Myrtaceae) na planície de alagável do Alto Rio Paraná, estados de Mato Grosso do Sul e Paraná, Brasil. Acta Botanica Brasilica 20: 529-548.

Santos, M.J.C. \& Paiva, S.N. 2002. Os sistemas agroflorestais como alternativa econômica em pequenas propriedades rurais: estudo de caso. Ciência Florestal 12: $135-141$.

Siebert, D.A., Bastos, J., Spudeit, D.A., Micke, G.A. \& Albertona, M.D. 2017. Determination of phenolic profile by HPLC-ESI-MS/MS and anti-inflammatory activity of crude hydroalcoholic extract and ethylacetate fraction from leaves of Eugenia brasiliensis. Revista Brasileira de Farmacognosia 27: 459-465.

Silva, C.V., Bilia, D.A.C. \& Barbedo, C.J. 2005. Fracionamento e germinação de sementes de Eugenia. Revista Brasileira de Sementes 27: 86-92.

Silva, E.A.A., Oliveira, J.M. \& Pereira, W.V.S. 2018. Fisiologia das sementes. In: Barbedo, C.J. \& Santos Junior, N.A. (orgs.) Sementes do Brasil: produção e tecnologia para espécies da flora brasileira. Instituto de Botânica, São Paulo. pp. 15-40.

Sosinski Junior, E.E., Urruth, L.M., Barbieri, R.L., Marchi, M.M. \& Martens, S.G. 2019. On the ecological recognition of Butia palm groves as integral ecosystems: why do we need to widen the legal protection and the in situ/ on-farm conservation approaches? Land Use Policy 81: 124-130.

Stavrinides, A., Dussert. S., Combes, M.C., FockBastide, I., Severac, D., Minier, J., Bastos-Siqueira, A., Demolombe, V., Hem, S., Lashermes, P. \& Joët, T. 2019. Seed comparative genomics in three coffee species identify desiccation tolerance mechanisms in intermediate seeds. Journal of Experimental Botany erz508.

Thomas, M., Dawson, J.C., Goldringer, I. \& Bonneuil, C. 2011. Seed exchanges, a key to analyze crop diversity dynamics in farmer-led on-farm conservation. Genet Resour Crop Evol 58: 321-338.

Tomielis, I.P., Guilherme, D.O., Brito, V.H.S., Maia, J.T.L.S. \& Cereda, M.P. 2016. Germination effect of different substrates on grumixama seeds. Nucleus 13: 185-188.

Tweddle, J.C., Dickie, J.B., Baskin, C.C. \& Baskin, J.M. 2003. Ecological aspects of seed desiccation sensitivity. Journal of Ecology 91: 294-304.

Villaça, I.de.M. \& Magenta, M.A.G. 2017. Plantas utilizadas pela população de Monte Cabrão, Santos, SP, Brasil. In: Anais do Encontro Nacional de PósGraduação - VI ENPG, Universidade Santa Cecília, Santos, SP. 1: pp. 286-290. 